\title{
NEF COTANGENT BUNDLES OF BRANCHED COVERINGS
}

\author{
MICHAEL J. SPURR
}

(Communicated by Louis J. Ratliff, Jr.)

\begin{abstract}
In a previous paper we characterized those $H(\Lambda, n)$ (compact complex surfaces constructed by Hirzebruch) that have nef cotangent bundle. In this article we extend the methods to study more general branched coverings with regard to nefness of their cotangent bundles.
\end{abstract}

\section{INTRODUCTION}

We first develop the notation. Let $\Lambda=\left\{L_{1}, \ldots, L_{k}\right\}$ be an arrangement of $k$ lines in $\mathbf{P}^{2}=\mathbf{P}^{2}(\mathbf{C})$. For $p \in \mathbf{P}^{2}, r_{p}$ is the number of lines in $\Lambda$ containing $p . \mathbf{B P}^{2}$ is the blowup of $\mathbf{P}^{2}$ at all $p$ with $r_{p} \geq 3$. Let $L_{j}^{\prime}$ denote the proper transform of $L_{j}$ in $\mathbf{B P}^{2}$ and $\Lambda^{\prime}=\bigcup\left\{L_{j}^{\prime}\right\}$. One defines $t_{j}$ by $t_{j}=$ cardinality $\left\{p \in \mathbf{P}^{2} \mid r_{p}=j\right\}$. Let $E_{i}$ denote the exceptional curve in $\mathbf{B P} \mathbf{P}^{2}$ over $p_{i}$ with $r_{p_{i}} \geq 3$, and let $\pi_{i}: \mathbf{B P}^{2} \rightarrow E_{i}$ be projection. For $\pi: X \rightarrow \mathbf{B P}^{2}$ a branched covering, $s_{i}: X \rightarrow S C_{i}$ will be the Stein factorization of $\pi_{i} \circ \pi: X \rightarrow E_{i}$.

For any vector bundle $E$ over a base manifold $M$, the projectivization $\mathbf{P}(E)$ is a fiber bundle over $M$, with fiber $\mathbf{P}_{q}(E)$ over $q \in M$ given by $\mathbf{P}_{q}(E) \approx\left(E_{q}^{*} \backslash 0\right) / \mathbf{C}^{*}$. There is a tautological line bundle $\xi_{E}$ over $\mathbf{P}(E)$ satisfying (i) $\left.\xi_{E}\right|_{\mathbf{P}_{q}(E)} \approx O(1)_{\mathbf{P}_{q}(E)} \forall q \in M$ and (ii) the projection $\rho_{E}: \mathbf{P}(E) \rightarrow M$ gives $\rho_{E^{*}}\left(\xi_{E}\right) \approx E$. In the case that $E=T^{*} M$ we will denote $\rho_{E}=\rho_{T^{*} M}$ simply by $\rho$.

Recall that the vector bundle $E$ is nef if $\xi_{E}$ over $\mathbf{P}(E)$ is nef, that is, $c_{1}\left(\xi_{E}\right) \cdot C \geq 0$ for all effective curves $C$ in $\mathbf{P}(E)$.

In what follows, $g(C)$ and $e(C)$ will denote the genus and euler number, respectively, of a curve $C$. For further development see $[4,5,1]$.

The problem approached here will be: given a branched covering $\pi: X \rightarrow Y$ of compact complex surfaces, find conditions on $Y$ along with the ramification set of $\pi$ in $X$ sufficient to guarantee that $T^{*} X$ is nef. Cases handled include: (i) $Y=\mathbf{B P}^{2}$ and (ii) $T^{*} Y$ is nef.

Received by the editors May 1, 1991 and, in revised form, September 10, 1991; presented to the American Mathematical Society Special Session on Algebraic Geometry, November 1990, Denton, Texas.

1991 Mathematics Subject Classification. Primary 14J60, 14E22, 14E20; Secondary 32J15, $32 \mathrm{C} 10$. 


\section{NeF COTANGENT BUNDLES}

In this section we prove the main theorems, including the following working theorem.

Theorem 1. Let $X$ and $Z$ be compact complex manifolds with $\operatorname{dim}_{\mathbf{C}} X=2$, $\operatorname{dim}_{\mathrm{C}} Z \geq 2$, and with $Z$ having nef cotangent bundle. Let $\pi: X \rightarrow Z$ be a holomorphic mapping with $X^{\prime}:=\left\{x \in X\left|\pi_{*}\right|_{x}\right.$ is not of maximal rank $\}$. Assume that $X^{\prime}=\bigcup B_{j} \cup q_{1} \cup q_{2} \cup \cdots \cup q_{N}$, where the $B_{j}$ are irreducible, smooth curves and the $q_{1}, q_{2}, \ldots, q_{N}$ are points. Then $X$ has nef cotangent bundle if $B_{j} \cdot B_{j} \leq 0$ and $e\left(B_{j}\right) \leq 0$ for each $B_{j}$ in $\bigcup B_{j}$.

Proof. Assume that $B_{j} \cdot B_{j} \leq 0$ and $e\left(B_{j}\right) \leq 0$ for each $B_{j}$ in $\bigcup B_{j}$. We prove nefness of $T^{*} X$.

The mapping $\pi: X \rightarrow Z$ induces $\pi_{*}: T X \rightarrow T Z$, which in turn induces $\Pi: \mathbf{P}\left(T^{*} X\right) \rightarrow \mathbf{P}\left(T^{*} Z\right)$. Let $\xi_{1}$ be the tautological bundle over $\mathbf{P}\left(T^{*} X\right)$, and let $\xi_{2}$ be the tautological bundle over $\mathbf{P}\left(T^{*} Z\right)$. $\Pi$ has indeterminacy set that is contained in $\rho^{-1}(A)$ where $\rho: \mathbf{P}\left(T^{*} X\right) \rightarrow X$ is projection and $A=\bigcup B_{j} \cup$ $q_{1} \cup \cdots \cup q_{N}$.

Let $C$ be an effective irreducible curve in $\mathbf{P}\left(T^{*} X\right)$. For $T^{*} X$ to be nef we show that $C \cdot \xi_{1}^{-1} \leq 0$. This is accomplished in three cases.

Case 1. Suppose that $\rho^{-1}(A) \not \supset C$. Let $\nu: \eta C \rightarrow C$ be the normalization of $C$. In this setting $\Pi \circ \nu: C \rightarrow \mathbf{P}\left(T^{*} Z\right)$ extends to be well defined and, by Lemma 2 , we have that $(\Pi \circ \nu)^{*}\left(\xi_{2}^{-1}\right) \cong \nu^{*}\left(\xi_{1}^{-1}\right)+D$ where $D$ is an effective divisor on $\eta C$. So

$$
\begin{aligned}
C \cdot \xi_{1}^{-1} & =\eta C \cdot \nu^{*}\left(\xi_{1}^{-1}\right)=\eta C \cdot\left((\Pi \circ \nu)^{*}\left(\xi_{2}^{-1}\right)-D\right) \\
& =\operatorname{deg}(\Pi \circ \nu)(\Pi \circ \nu)(C) \cdot \xi_{2}^{-1}-\eta C \cdot D \leq 0 .
\end{aligned}
$$

The last inequality follows since $\Pi \circ \nu(C)$ is a curve in $\mathbf{P}\left(T^{*} Z\right), \xi_{2}$ is nef, and $D$ is effective on $\eta C$.

Case 2. Suppose that $C$ is a fiber of $\rho$ (and therefore $\left.C \cong \mathbf{P}^{\mathbf{1}}\right)$. Then $C \cdot \xi_{1}^{-1}=-1$ since $\left.\xi_{1}\right|_{C} \cong O(1)$.

Case 3. Suppose $C$ is contained in $\rho^{-1}(A)$ but is not a fiber of $\rho$. Then $\rho(C)=B_{j}$ for some $j$. For $\nu: \eta C \rightarrow C$ the normalization of $C$, one has that $\rho \circ \nu: \eta C \rightarrow \rho(C)$. One has the vector bundle maps

$$
0 \rightarrow \nu^{*}\left(\left.\xi_{1}^{-1}\right|_{C}\right) \rightarrow \nu^{*} \rho^{*}\left(\left.T X\right|_{\rho(C)}\right)
$$

and

$$
0 \rightarrow \nu^{*} \rho^{*} T \rho(C) \rightarrow \nu^{*} \rho^{*}\left(\left.T X\right|_{\rho(C)}\right) \rightarrow \nu^{*} \rho^{*} N \rho(C) \rightarrow 0 .
$$

Hence one of the sequence of sheaves (2.1) or (2.2) must be valid.

$$
\begin{aligned}
& 0 \rightarrow \nu^{*} \xi_{1}^{-1} \rightarrow \nu^{*} \rho^{*} T \rho(C) \rightarrow Z_{1} \rightarrow 0, \\
& 0 \rightarrow \nu^{*} \xi_{1}^{-1} \rightarrow \nu^{*} \rho^{*} N \rho(C) \rightarrow Z_{2} \rightarrow 0
\end{aligned}
$$

where $Z_{1}$ and $Z_{2}$ are sheaves with finite support on $\eta C$. By letting $M_{1}=$ $\nu^{*} \rho^{*} T \rho(C)$ and $M_{2}=\nu^{*} \rho^{*} N \rho(C)$, we rewrite (2.1) and (2.2) as

$$
0 \rightarrow \nu^{*} \xi_{1}^{-1} \rightarrow M_{i} \rightarrow Z_{i} \rightarrow 0 \text {. }
$$


By utilizing the long exact sequence associated to (2.3), along with RiemannRoch, one concludes that

$$
c_{1}\left(\xi_{1}^{-1}\right) \cdot C=c_{1}\left(\nu^{*} \xi_{1}^{-1}\right) \cdot \eta C \leq c_{1}\left(M_{i}\right) \cdot \eta C=\left\{\begin{array}{l}
\operatorname{deg}(\rho \circ \nu) c_{1}(T \rho(C)) \cdot \rho(C), \\
\operatorname{deg}(\rho \circ \nu) c_{1}(N \rho(C)) \cdot \rho(C) .
\end{array}\right.
$$

Now $c_{1}(T \rho(C)) \cdot \rho(C)=e(\rho(C))=e\left(B_{j}\right) \leq 0$ by our hypothesis. And similarly we have that $c_{1}\left(N(\rho(C)) \cdot \rho(C)=\rho(C) \cdot \rho(C)=B_{j} \cdot B_{j} \leq 0\right.$ by hypothesis. Therefore, in all cases $\xi_{1}^{-1} \cdot C \leq 0$. So $\xi_{1} \cdot C \geq 0$ and $\xi_{1}$ is nef, giving that $T^{*} X$ is nef.

In the setting of Theorem 1 we prove

Lemma 2. For $C$ contained in $\mathbf{P}\left(T^{*} X\right)$ with $C$ not contained in $\rho^{-1}(A)$, let $\nu: \eta C \rightarrow C$ be normalization. Then

$$
(\Pi \circ \nu)^{*}\left(\xi_{2}^{-1}\right) \cong \nu^{*} \xi_{1}^{-1}+D
$$

where $D$ is an effective divisor on $\eta C$.

Proof. First note that if $g: L_{1} \rightarrow L_{2}$ is a holomorphic mapping of complex line bundles over a curve (which we take to be) $\eta C$, then $L_{2} \cong L_{1}+D_{g}$ where $D_{g}$ is the effective divisor on $\eta C$ induced by the vanishing of the mapping $g$. Next we let $L_{1}=\nu^{*} \xi_{1}^{-1}$ and $L_{2}=(\Pi \circ \nu)^{*}\left(\xi_{2}^{-1}\right)$ where $\Pi \circ \nu$ extends to be well defined on $\eta C$ as $C$ is not contained in $\rho^{-1}(A)$. Observe that $\pi_{*}: T X \rightarrow T Z$ induces the mapping $\pi_{*}: \xi_{1}^{-1} \rightarrow \xi_{2}^{-1}$, which in turn induces $\pi_{*}: \nu^{*} \xi_{1}^{-1} \rightarrow(\Pi \circ \nu)^{*}\left(\xi_{2}^{-1}\right)$. Finally we take $g$ to be the mapping $\pi_{*}: \nu^{*} \xi_{1}^{-1} \rightarrow$ $(\Pi \circ \nu)^{*}\left(\xi_{2}^{-1}\right)$, giving the lemma with $D:=D_{g}$.

Theorem 3. Let $\Lambda$ be an arrangement of $k \geq 3$ lines in $\mathbf{P}^{2}$ with $t_{k}=0$. Assume there are at least two points $p_{i}, i=1,2$, with $r_{p_{i}} \geq 3$. Let $\mathbf{B P}^{2}$ be the blowup of $\mathbf{P}^{2}$ at each point $p_{j}$ with $r_{p_{j}} \geq 3$, and let $E_{j}$ be the exceptional curve over $p_{j}$ with $\pi_{j}: \mathbf{B P}^{2} \rightarrow E_{j}$ projection. Let $L$ be the line containing $p_{1}$ and $p_{2}$ in $\mathbf{P}^{2}$, and let $L^{\prime}$ be its proper transform in $\mathbf{B P}^{2}$.

Let $\pi: X \rightarrow \mathbf{B P}^{2}$ be any branched covering of $\mathbf{B P}^{2}$ with branch locus contained in $\Lambda^{\prime} \cup E_{1} \cup \cdots \cup E_{n} \cup L^{\prime}$ where $\pi^{-1}\left(\Lambda^{\prime} \cup E_{1} \cup \cdots \cup E_{n} \cup L^{\prime}\right)$ has irreducible components that are smooth. If $\pi: X \rightarrow \mathbf{B P}^{2}$ satisfies

(1) For each $L_{\alpha} \in \Lambda \cup\{L\}, e\left(\widehat{L}_{\alpha}\right) \leq 0$, and $\widehat{L}_{\alpha} \cdot \widehat{L}_{\alpha} \leq 0$ for each irreducible component $\widehat{L}_{\alpha}$ of $\pi^{-1}\left(L_{\alpha}^{\prime}\right)$;

(2) $e\left(C_{j}\right) \leq 0$ for each irreducible component $C_{j}$ of $\pi^{-1}\left(E_{j}\right), j=1, \ldots, n$;

(3) $g\left(S C_{i}\right) \geq 1$ for $i=1,2$ where $s_{i}: X \rightarrow S C_{i}$ and $\rho_{i}: S C_{i} \rightarrow E_{i}$ is the Stein factorization of $\pi_{i} \circ \pi: X \rightarrow E_{i}$;

then $T^{*} X$ is nef.

Proof. Define $Z=S C_{1} \times S C_{2}$ and construct the mapping $s: X \rightarrow Z$ given by $s=s_{1} \times s_{2}$ where $s_{i}: X \rightarrow S C_{i}$ is the Stein factorization of $\pi_{i} \circ \pi: X \rightarrow E_{i}$. Then since $\left(\pi_{1} \times \pi_{2}\right) \circ \pi=\left(\rho_{1} \times \rho_{2}\right) \circ\left(s_{1} \times s_{2}\right)$ and $\pi_{1} \times \pi_{2}: \mathbf{B P}^{2} \rightarrow E_{1} \times E_{2}$ is biholomorphic on $\mathbf{B P}^{2} \backslash \cup E_{1} \cup \cdots \cup E_{n} \cup L^{\prime}$, the singular set of $s_{1} \times s_{2}$ is contained in the set $\pi^{-1}\left(\Lambda^{\prime} \cup E_{1} \cup \cdots \cup E_{n} \cup L^{\prime}\right)=\bigcup \widehat{L}_{j} \cup \pi^{-1}\left(E_{1}\right) \cup \cdots \cup \pi^{-1}\left(E_{n}\right) \cup \pi^{-1}\left(L^{\prime}\right)$.

By hypotheses (1) and (2), $e\left(B_{j}\right) \leq 0$ for each $B_{j}$ that is contained in the set $\bigcup \widehat{L}_{j} \cup \pi^{-1}\left(E_{1}\right) \cup \cdots \cup \pi^{-1}\left(E_{n}\right) \cup \pi^{-1}\left(L^{\prime}\right)$. (1) gives that $B_{j} \cdot B_{j} \leq 0$ for $B_{j}$ contained in the set $\cup \widehat{L}_{j} \cup \pi^{-1}\left(L^{\prime}\right)$. If $B_{j}$ is contained in $\pi^{-1}\left(E_{1}\right) \cup \cdots \cup \pi^{-1}\left(E_{n}\right)$ 
then $B_{j} \cdot B_{j} \leq 0$, since if $n_{j}$ is the branching order of $\pi$ along $B_{j}$ then $B_{j} \cdot B_{j} \leq\left(1 / n_{j}\right) \operatorname{deg}\left(\left.\pi\right|_{B_{j}}\right) E_{j}^{2}<0$. Now (3) gives that $T^{*} Z$ is nef. Apply Theorem 1 to the mapping $s: X \rightarrow Z$ to conclude that $T^{*} X$ is nef.

Remark. One can drop the extra hypotheses on $L$ (the line of collinearity containing $p_{1}$ and $p_{2}$ ) of Theorem 3 and still conclude that $T^{*} X$ is nef, provided that there is a third point $p_{3}$, noncollinear with $p_{1}$ and $p_{2}$, also satisfying $g\left(S C_{3}\right) \geq 1$ where $s_{3}: X \rightarrow S C_{3}$ is the Stein factorization of $\pi_{3} \circ \pi: X \rightarrow$ $E_{3}$. The proof is similar to that of Theorem 3 if one lets $s$ be the mapping $s_{1} \times s_{2} \times s_{3}: X \rightarrow S C_{1} \times S C_{2} \times S C_{3}$.

Theorem 4. Let $X$ and $Y$ be compact complex surfaces with $Y$ having nef cotangent bundle, and let $\pi: X \rightarrow Y$ be a branched covering with ramification set $\cup B_{j}$ in $X$, where we assume that the $B_{j}$ are irreducible and smooth. Let $\cup C_{\alpha}$ be the branch locus of $\pi$ in $Y$. Then

$$
\begin{aligned}
C_{\alpha} \cdot & C_{\alpha} \leq 0 \text { for each } C_{\alpha} \text { in the branch locus in } Y \\
& \Rightarrow B_{j} \cdot B_{j} \leq 0 \text { for each } B_{j} \text { in the ramification set in } X \\
& \Rightarrow X \text { has nef cotangent bundle. }
\end{aligned}
$$

Proof. Assume that $C_{\alpha} \cdot C_{\alpha} \leq 0$ for each $C_{\alpha}$ in the branch locus in $Y$. Let $\pi^{*}\left(C_{\alpha}\right)=\sum_{k} n_{\alpha k} B_{\alpha k}$. Then

$$
\begin{aligned}
B_{\alpha j} \cdot \pi^{*}\left(C_{\alpha}\right) & =B_{\alpha j} \cdot \sum_{k} n_{\alpha k} B_{\alpha k}=n_{\alpha j} B_{\alpha j} \cdot B_{\alpha j}+B_{\alpha j} \cdot \sum_{k \neq j} n_{\alpha k} B_{\alpha k} \\
& =\operatorname{deg}\left(\left.\pi\right|_{B_{\alpha j}}\right) C_{\alpha} \cdot C_{\alpha}
\end{aligned}
$$

whence

$$
B_{\alpha j} \cdot B_{\alpha j}=n_{\alpha j}^{-1} \operatorname{deg}\left(\left.\pi\right|_{B_{\alpha j}}\right) C_{\alpha} \cdot C_{\alpha}-n_{\alpha j}^{-1} B_{\alpha j} \cdot \sum_{k \neq j} n_{\alpha k} B_{\alpha k} \leq 0,
$$

giving the first implication.

Assume that $B_{j} \cdot B_{j} \leq 0$ for each $B_{j}$ in the ramification set. We prove nefness of $T^{*} X$. Observe that for each $B_{j}$ in the ramification set $e\left(B_{j}\right) \leq 0$, otherwise $e\left(\pi\left(B_{j}\right)\right)>0$ in $Y$, which contradicts that $T^{*} Y$ is nef. Apply Theorem 1 to $\pi: X \rightarrow Y$ to conclude that $T^{*} X$ is nef.

Next we extend our viewpoint to arrangements of pencils of curves.

Definition. Let $P_{i}$ be a pencil of curves in $\mathbf{P}^{2}$ and $\Lambda_{i}=\left\{C_{i j} \mid j=1, \ldots, k_{i}\right\}$ be an arrangement of smooth curves in $P_{i}$ with each $C_{i j}$ intersecting each $C_{i J}$ transversely (only) in the base locus (consisting of isolated points). We label the base locus of $P_{i}$ by $\mathrm{BL} P_{i}$.

(1) An arrangement of pencils $\Lambda$ is defined to be $\Lambda=\bigcup \Lambda_{i}$ with each $\Lambda_{i}$ as above.

(2) A general arrangement of pencils is defined to be an arrangement of pencils $\Lambda=\bigcup \Lambda_{i}$ satisfying:

(a) $\mathrm{BLP}_{i} \cap \mathrm{BL} P_{j}=\varnothing$ if $i \neq j$.

(b) After blowup of all points in $U B L P_{i}$ to get $\mathbf{B P}^{2}$ and letting $E C$ be the resulting set of exceptional curves and $\Lambda^{\prime}$ be the arrangement of proper transforms $C_{i j}^{\prime}$ of the curves $C_{i j}$ in $\Lambda$, we have that $E C \cup \Lambda^{\prime}$ consists of smooth curves meeting transversely in normal crossings. 
(c) For each $i$ and each $j, C_{i j}^{\prime}$ is a nonsingular fiber of $\pi_{i}$.

(d) There are $k \geq 3$ pencils, say $P_{1}, P_{2}, \ldots, P_{k}$, with $\Lambda \supset \Lambda_{1} \cup \Lambda_{2} \cup \cdots \cup \Lambda_{k}$ satisfying that $\pi_{1} \times \pi_{2} \times \cdots \times \pi_{k}: \mathbf{B P}^{\mathbf{2}} \rightarrow \mathbf{P}^{\mathbf{1}} \times \mathbf{P}^{\mathbf{2}} \times \cdots \mathbf{P}^{\mathbf{1}}$ is of maximal rank 2 on $\mathbf{B P}^{2} \backslash E C$ except possibly at finitely many points $q_{1}, q_{2}, \ldots, q_{N}$. Here each $\pi_{i}: \mathbf{B P}^{2} \rightarrow \mathbf{P}^{1}$ is the holomorphic mapping associated to the pencil $P_{i}$.

(e) For all $i$, no point of $C_{i j}$ lies in the base locus of $P_{I}$ for $I \neq i$.

Theorem 5. Let $\Lambda$ be a general arrangement of pencils in $\mathbf{P}^{2}$. Blowup all points in the base loci to obtain $\mathbf{B P}^{2}$. Let $\pi: X \rightarrow \mathbf{B P}^{2}$ be any branched covering of $\mathbf{B P}^{2}$ with branch locus in $\mathbf{B P}^{2}$ contained in $E C \cup \Lambda^{\prime}$ and ramification set in $\pi^{-1}\left(E C \cup \Lambda^{\prime}\right)$ having irreducible components that are smooth.

Let $\pi_{i}: \mathbf{B P}^{\mathbf{2}} \rightarrow \mathbf{P}^{\mathbf{1}}$ be the natural holomorphic projection associated to each pencil $P_{i}$. Let $s_{i}: X \rightarrow S C_{i}$ and $\rho_{i}: S C_{i} \rightarrow \mathbf{P}^{\mathbf{1}}$ be the Stein factorization of $\pi_{i} \circ \pi: X \rightarrow \mathbf{P}^{1}$. Then $T^{*} X$ is nef if:

(1) For the projections $\pi_{1}, \ldots, \pi_{k}$ with $\pi_{1} \times \pi_{2} \times \cdots \times \pi_{k}: \mathbf{B P}^{\mathbf{2}} \rightarrow \mathbf{P}^{\mathbf{1}} \times \mathbf{P}^{\mathbf{1}} \times$ $\cdots \times \mathbf{P}^{\mathbf{1}}$ of maximal rank on $\mathbf{B P}^{\mathbf{2}} \backslash\{E C \cup$ finitely many points $\}$, we have that the euler numbers $e\left(S C_{i}\right) \leq 0$ for $i=1, \ldots, k$.

(2) For each $C_{j} \in E C \cup \Lambda^{\prime}$ and each irreducible component $\widehat{C}_{j}$ of $\pi^{-1}\left(C_{j}\right)$ one has that $e\left(\widehat{C}_{j}\right) \leq 0$ and $\widehat{C}_{j} \cdot \widehat{C}_{j} \leq 0$.

Proof. Define $Z=S C_{1} \times S C_{2} \times \cdots \times S C_{k}$ and construct the mapping $s: X \rightarrow Z$ given by $s=s_{1} \times s_{2} \times \cdots \times s_{k}$ where $s_{i}: X \rightarrow S C_{i}$ is the Stein factorization of $\pi_{i} \circ \pi: X \rightarrow \mathbf{P}^{1}$. Then since $\left(\pi_{1} \times \pi_{2} \times \cdots \times \pi_{k}\right) \circ \pi=\left(\rho_{1} \times \rho_{2} \times \cdots \times\right.$ $\left.\rho_{k}\right) \circ\left(s_{1} \times s_{2} \times \cdots \times s_{k}\right)$, the singular set of $s_{1} \times s_{2} \times \cdots \times s_{k}$ is contained in the singular set of $\left(\pi_{1} \times \pi_{2} \times \cdots \times \pi_{k}\right) \circ \pi \cup \pi^{-1}(E C)$. Therefore the singular set of $s_{1} \times s_{2} \times \cdots \times s_{k}$ is contained in $\pi^{-1}\left(q_{1} \cup \cdots \cup q_{N}\right) \cup\left(\cup \widehat{C}_{j}\right)$. (1) gives that $T^{*} Z$ is nef and (2) allows the application of Theorem 1 to conclude that $T^{*} X$ is nef.

Remark. See Example 4.4 for a construction of a class of surfaces to which we apply Theorem 5 .

\section{Applications}

As an application of Theorem 3 we study galois branched coverings. Recall that a branched covering $\pi: X \rightarrow Y$ is galois if the deck transformations act transitively on fibers of $\pi$.

We begin by setting up the notation. Let $\Lambda$ be an arrangement of lines in $\mathbf{P}^{2}$ with at least 2 points $p_{1}$ and $p_{2}$ having $r_{p_{i}} \geq 3$ for $i=1,2 . \mathbf{B P}^{2}$ is the blowup of $\mathbf{P}^{2}$ at each point $p_{j}$ with $r_{p_{j}} \geq 3$, and let $E_{j}$ be the exceptional curve over $p_{j}$. Let $L$ be the line containing $p_{1}$ and $p_{2}$ in $\mathbf{P}^{2}$, and let $L^{\prime}$ be its proper transform in $\mathbf{B P}^{2}$.

Let $\pi: X \rightarrow \mathbf{B P}^{2}$ be a galois branched covering that is locally of form $(u, v) \rightarrow\left(u^{n}, v^{m}\right)$ with branch locus in $\mathbf{B P}^{2}$ contained in $\Lambda^{\prime} \cup\left(\cup E_{j}\right)$. The line $L_{i}^{\prime} \in \Lambda^{\prime}$ is assigned branching order $n_{i}$, where $n_{i}$ is the branching order for each component $\widehat{L}_{i}^{\prime}$ of $\pi^{-1}\left(L_{i}^{\prime}\right) . E_{j}$ is similarly assigned branching order $m_{j}$, where $m_{j}$ is the branching order for each component $\widehat{E}_{j}$ of $\pi^{-1}\left(E_{j}\right)$.

Let the five parameter classes be given as follows:

(i) $\alpha$ parametrizes $L_{\alpha}^{\prime} \in \Lambda^{\prime} \cup\left\{L^{\prime}\right\}$;

(ii) $i$ parametrizes $L_{i}^{\prime} \in \Lambda^{\prime}$; 
(iii) $j$ parametrizes $E_{j} \in E C:=$ the set of exceptional curves in $\mathbf{B P}^{2}$;

(iv) given $\alpha, \beta$ parametrizes $p_{\beta} \in P L_{\alpha}$ where $P L_{\alpha}:=\left\{p_{\beta} \in L_{\alpha}^{\prime} \mid \exists\right.$ two lines $L_{1}^{\prime}, L_{2}^{\prime} \in \Lambda^{\prime} \backslash L_{\alpha}^{\prime}$ with $\left.L_{1}^{\prime} \cap L_{2}^{\prime}=p_{\beta}\right\}$

(v) given $\alpha, \gamma$ parametrizes $p_{\gamma} \in Q L_{\alpha}$ where

$$
Q L_{\alpha}:=\left\{p_{\gamma} \in L_{\alpha}^{\prime} \mid \exists i \text { with } i \neq \alpha \text { with } p_{\gamma} \in L_{i}^{\prime} \cap L_{\alpha}^{\prime} \text { and } p_{\gamma} \notin P L_{\alpha}\right\} \text {. }
$$

For $p_{\gamma} \in Q L_{\alpha}$ with $p_{\gamma}=L_{i}^{\prime} \cap L_{\alpha}^{\prime}$ we let $n_{\gamma}:=n_{i}$. For $p_{\beta} \in P L_{\alpha}$ with $p_{\beta}=L_{i}^{\prime} \cap L_{I}^{\prime}$ let

$$
M_{\beta}:=\text { the least common multiple }\left(n_{i}, n_{I}\right)
$$

where $n_{i}$ and $n_{I}$ are the respective branching orders of $L_{i}^{\prime}$ and $L_{I}^{\prime}$.

Theorem 6. Let $\Lambda$ be an arrangement of lines in $\mathbf{P}^{2}$ with at least two points $p_{1}$ and $p_{2}$ having $r_{p_{i}} \geq 3$ for $i=1,2$. Let $L$ be the line containing $p_{1}$ and $p_{2}$ in $\mathbf{P}^{2}$, and let $L^{\prime}$ be its proper transform in $\mathbf{B P}^{\mathbf{2}}$. Let $\pi: X \rightarrow \mathbf{B P}^{\mathbf{2}}$ be a galois branched covering that is locally of form $(u, \nu) \rightarrow\left(u^{n}, \nu^{m}\right)$ with branch locus in BP $^{2}$ contained in $\Lambda^{\prime} \cup\left(\cup E_{j}\right)$ and with $\pi^{-1}\left(\Lambda^{\prime} \cup\left(\cup E_{j}\right) \cup L^{\prime}\right)$ having irreducible components that are smooth. Then, in the notation of the above paragraphs, $T^{*} X$ is nef if

$$
\text { (1) } 2-\sum_{p_{\gamma} \in Q L_{\alpha}}\left(1-\frac{1}{n_{\gamma}}\right)-\sum_{p_{\beta} \in P L_{\alpha}}\left(1-\frac{1}{M_{\beta}}\right)-\sum_{j}\left(1-\frac{1}{m_{j}}\right) E_{j} \cdot L_{\alpha}^{\prime} \leq 0
$$

for each $L_{a} \in \Lambda \cup\{L\}$.

(2) $2-\sum_{i}\left(1-1 / n_{i}\right) E_{j} \cdot L_{i}^{\prime} \leq 0$ for each $E_{j}$ an exceptional curve.

(3) Each $L_{j}$ in $\Lambda$ is blown up at least once.

(4) $g\left(S C_{i}\right)$ is positive for $i=1,2$ where $s_{i}: X \rightarrow S C_{i}$ is the Stein factorization of $\pi_{i} \circ \pi: X \rightarrow \mathbf{P}^{1}$.

Proof. This is a direct result of Theorem 3 after one computes that

$$
\begin{aligned}
& e\left(\widehat{L}_{\alpha}^{\prime}\right)=\left(\left.\operatorname{deg} \pi\right|_{\widehat{L}_{\alpha}^{\prime}}\right)(2-\sum_{p_{\gamma} \in Q L_{\alpha}}\left(1-\frac{1}{n_{\gamma}}\right) \\
&\left.\quad-\sum_{p_{\beta} \in P L_{\alpha}}\left(1-\frac{1}{M_{\beta}}\right)-\sum_{j}\left(1-\frac{1}{m_{j}}\right) E_{j} \cdot L_{\alpha}^{\prime}\right), \\
& e\left(\widehat{E}_{j}\right)=\left(\left.\operatorname{deg} \pi\right|_{\widehat{E}_{j}}\right)\left(2-\sum_{i}\left(1-\frac{1}{n_{i}}\right) E_{j} \cdot L_{i}^{\prime}\right),
\end{aligned}
$$

and

$$
\widehat{L}_{\alpha}^{\prime} \cdot \widehat{L}_{\alpha}^{\prime} \leq \frac{1}{n_{\alpha}} \operatorname{det}\left(\left.\pi\right|_{\widehat{L}_{\alpha}^{\prime}}\right) L_{\alpha}^{\prime} \cdot L_{\alpha}^{\prime} \quad \text { as in (2.4). }
$$

Here $n_{\alpha}=1$ if $L_{\alpha}=L$ and $L \notin \Lambda$. See Höfer's dissertation [3] for similar calculations. The two euler numbers are negative by (1) and (2) of the theorem. $L_{\alpha}^{\prime} \cdot L_{\alpha}^{\prime} \leq 0$ by (3) and the fact that $L^{\prime}$ has been blown up at least twice.

Remark. There are many situations where hypothesis (4) of Theorem 6 holds naturally. See Example 4.1.

We give similarly an application of Theorem 4 in the galois setting. 
Theorem 7. Let $T^{*} Y$ be nef and let $\pi: X \rightarrow Y$ be a galois branched covering having ramification set $\bigcup B_{j}$ in $X$ and branch locus $\bigcup C_{\alpha}$ in $Y$. We assume that the $B_{j}$ are smooth and meet transversely in at most normal crossings and that the $C_{\alpha}$ are smooth. We further assume that $\pi$ can be locally represented by coordinate charts of the form $(u, \nu) \rightarrow\left(u^{n}, \nu^{m}\right)$. Then

$$
\begin{aligned}
& C_{\alpha} \cdot C_{\alpha} \leq 0 \text { for each } C_{\alpha} \text { in the branch locus in } Y \\
& \Leftrightarrow B_{j} \cdot B_{j} \leq 0 \text { for each } B_{j} \text { in the ramification set in } X \\
& \Leftrightarrow X \text { has nef cotangent bundle. }
\end{aligned}
$$

Proof. Under the assumptions that $\pi$ is galois and locally of form $(u, v) \rightarrow$ $\left(u^{n}, v^{m}\right)$ and the $C_{\alpha}$ are smooth, it cannot happen that $B_{\alpha j}$ meets $B_{\alpha k}$ for $j \neq k$ where $B_{\alpha j}$ and $B_{\alpha k}$ are irreducible components of $\pi^{*}\left(C_{\alpha}\right)$. (If $p \in$ $B_{\alpha j} \cap B_{\alpha k}$ and $(u, v)$ is a coordinate chart centered at $p$ with $\pi$ of form $(u, v) \rightarrow\left(u^{n}, v^{m}\right)=:(A, B)$, then the only local branching occurs at $u=0$ and $v=0$. Thus $B_{\alpha j}$ is given locally by, say, $u=0$ and then $B_{\alpha k}$ is given by $v=0$ (and on our chart $n=m)$. Then $\pi(\{u=0\} \cup\{v=0\})=\{A=$ $0\} \cup\{B=0\}$, which contradicts that $C_{\alpha}$ is smooth.) Hence (2.4) gives that $B_{\alpha j} \cdot B_{\alpha j}=n_{\alpha j}^{-1} \operatorname{deg}\left(\left.\pi\right|_{B_{\alpha j}}\right) C_{\alpha} \cdot C_{\alpha}$. So in this setting $B_{\alpha j} \cdot B_{\alpha j} \leq 0$ if and only if $C_{\alpha} \cdot C_{\alpha} \leq 0$, giving the first equivalence.

Theorem 4 gives that if $B_{j} \cdot B_{j} \leq 0$ for all curves $B_{j}$ in the ramification set then $T^{*} X$ is nef. It only remains to show the converse. We adapt a splitting lemma of Sommese [4]: if $B_{j}$ is smooth and contained in the ramification set of $\pi$, then there is a splitting $\left.T B_{j} \oplus N B_{j} \approx T X\right|_{B_{j}}$. The splitting is obtained by utilizing the natural sequence $\left.0 \rightarrow T B_{j} \rightarrow T X\right|_{B_{j}} \rightarrow N B_{j} \rightarrow 0$ and producing a sub line bundle $L$ of $\left.T X\right|_{B_{j}}$ that projects onto $N B_{j} . L$ is the unique line bundle contained in the annihilator of $\pi^{*} d A=n u^{n-1} d u$ and $\pi^{*} d B=$ $m v^{m-1} d v$. Here $(u, v)$ are local coordinates in $X$ with $B_{j}$ being given locally by $u=0$, and $(A, B)$ are local coordinates in $Y$ with $A=u^{n}$ and $B=v^{m}$. Once we have this splitting, $B_{j}$ and $N B_{j}$ determine a curve $C$ in $\mathbf{P}\left(T^{*} X\right)$ and $\xi_{1}^{-1} \cdot C=N B_{j} \cdot B_{j}=B_{j} \cdot B_{j} \leq 0$ since $\xi_{1} \cdot C$ is nonnegative by our assumption that $T^{*} X$ is nef.

\section{EXAMPLES}

Example 4.1. In [5], $H(\Lambda, n)$ with $k \geq 3$ was shown to have nef cotangent bundle iff

(a) For each $L \in \Lambda, L$ is blown up at least once (in obtaining $\mathbf{B P}^{2}$ ).

(b) If $n=2$ then $t_{3}=\#\left\{p \mid r_{p}=3\right\}=0$.

(c) For all $L \in \mathbf{P}^{2}, \delta(L) \neq 2$, and if $n=2$ then $\delta(L) \neq 3$ where $\delta(L)$ is defined as the cardinality of the branch locus in $L^{\prime}$ of $\left.\pi\right|_{\pi^{-1}\left(L^{\prime}\right)}: \pi^{-1}\left(L^{\prime}\right) \rightarrow L^{\prime}$.

Theorem 6 gives the sufficiency of (a), (b), and (c) for nefness of $T^{*} H(\Lambda, n)$. This follows since $H(\Lambda, n)$ is a galois branched covering of $\mathbf{B P}^{2}$, and one has in this setting that all branching orders are $n$. Thus

$$
2-\sum_{i}\left(1-\frac{1}{n_{i}}\right) E_{j} \cdot L_{i}^{\prime}=2-\left(1-\frac{1}{n}\right)\left(\sum_{i} E_{j} \cdot L_{i}^{\prime}\right)=2-\left(1-\frac{1}{n}\right) r_{p_{j}},
$$


and so Theorem $6(2)$ holds iff $2-(1-1 / n) r_{p_{j}} \leq 0$ iff (b) holds. Similarly one has that

$$
\begin{aligned}
2- & \sum_{p_{\gamma} \in Q L_{\alpha}}\left(1-\frac{1}{n_{\gamma}}\right)-\sum_{p_{\beta} \in P L_{\alpha}}\left(1-\frac{1}{M_{\beta}}\right)-\sum_{j}\left(1-\frac{1}{m_{j}}\right) E_{j} \cdot L_{\alpha}^{\prime} \\
& =2-\sum_{p_{\gamma} \in Q L_{\alpha}}\left(1-\frac{1}{n}\right)-\sum_{p_{\beta} \in P L_{\alpha}}\left(1-\frac{1}{n}\right)-\sum_{j}\left(1-\frac{1}{n}\right) E_{j} \cdot L_{\alpha}^{\prime} \\
& =2-\left(1-\frac{1}{n}\right)\left(\# Q L_{\alpha}+\# P L_{\alpha}+\sum_{j} E_{j} \cdot L_{\alpha}^{\prime}\right)=2-\left(1-\frac{1}{n}\right) \delta\left(L_{\alpha}\right),
\end{aligned}
$$

and so Theorem $6(1)$ holds iff $2-(1-1 / n) \delta\left(L_{\alpha}\right) \leq 0$ iff (under the assumption that (b) holds) (c) holds. Clearly Theorem 6(3) is the same condition as (a) above. Finally Theorem 6(4) holds automatically by the fact that each component of $\pi^{-1}\left(E_{j}\right)$ is a section of $s_{j}: H(\Lambda, n) \rightarrow S C_{j}$ (see [4]) so $g\left(\widehat{E}_{j}\right)=s\left(S C_{j}\right)>0$ by (b).

We remark that conditions (1), (2) of Theorem 6 are necessary for nefness of $T^{*} X$. Since for $H(\Lambda, n)$ (4) holds automatically, and since for $H(\Lambda, n)$, $\widehat{L}_{\alpha}^{\prime} \cdot \widehat{L}_{\alpha}^{\prime}=\left(1 / n_{\alpha}\right) \operatorname{deg}\left(\left.\pi\right|_{\widehat{L}_{\alpha}^{\prime}}\right) L_{\alpha}^{\prime} \cdot L_{\alpha}^{\prime}$, we have our equivalence.

Example 4.2. In [4] Sommese proves density of the Chern ratios $c_{1}^{2}(S) / c_{2}(S)$ in the interval $[1 / 5,3]$ for the class of minimal compact complex surfaces $S$ of general type. His method of exhibiting density in the interval $[2,3]$ relies on Hirzebruch's surface $H(\Lambda, 5)$ where $\Lambda$ is the $A_{1}(6)$ arrangement of lines in $\mathbf{P}^{2}$ (see [2, 4]). One has $c_{1}^{2}(H(\Lambda, 5))=3 c_{2}(H(\Lambda, 5))$ and also that there is a fibering $f: H(\Lambda, 5) \rightarrow C$ where $C$ is a Riemann surface of genus $g(C)=6$. For any branched covering $F: C^{\prime} \rightarrow C$, having branch locus in $C$ that is disjoint from the image in $C$ under $f$ of the set where $f$ is not of maximal rank, Sommese shows that the surfaces $S=F^{*}(H(\Lambda, 5))=C^{\prime} \times_{C} H(\Lambda, 5)$ have Chern ratios $c_{1}^{2}(S) / c_{2}(S)$ dense in the interval $[2,3] . H(\Lambda, 5)$ has ample cotangent bundle [4], and $F^{*}(H(\Lambda, 5))$ branch covers $H(\Lambda, 5)$ with ramification set in $F^{*}(H(\Lambda, 5))$ consisting of the fibers $B_{j}$ over the ramification set in $C^{\prime}$. Each $B_{j}$ has genus 76 [4], and clearly $B_{j} \cdot B_{j}=0$. By Theorem 4 the surfaces $F^{*}(H(\Lambda, 5))$ have nef cotangent bundle. However, $T^{*} F^{*}(H(\Lambda, 5))$ is not ample as is easily seen. First note that $\left.T F^{*}(H(\Lambda, 5))\right|_{B_{j}} \approx T B_{j} \oplus N B_{j}$. Let $R$ be the curve in $\mathbf{P}\left(T^{*} F^{*}(H(\Lambda, 5))\right.$ corresponding to $B_{j}$ and $N B_{j}$. Then for $\xi=\xi_{T^{*} F^{*}(H(\Lambda, 5))}$ one has that $\xi^{-1} \cdot R=N B_{j} \cdot B_{j}=B_{j} \cdot B_{j}=0$. Thus $\xi \cdot R=0$, giving that $T^{*} F^{*}(H(\Lambda, 5))$ is not ample. This gives a class of surfaces $X$ with $T^{*} X$ nef but not ample, and $c_{1}^{2}(X) / c_{2}(X)$ is dense in the interval $[2,3]$.

Example 4.3. The $F^{*}(H(\Lambda, 5))$ of Example 4.2 can also be seen as anple of Theorem 3. $F^{*}(H(\Lambda, 5))$ branch covers $H(\Lambda, 5)$ that in turn branch covers $\mathbf{B P}^{2}$. Since $F: C^{\prime} \rightarrow C$ need not be galois, $F^{*}(H(\Lambda, 5))$ need not be galois over $\mathbf{B P}^{2}$. But Theorem 3 is applicable in this setting. One checks from the facts given for $H(\Lambda, 5)$ in Examples 4.2 that Theorem 3(1)-(3) hold and $T^{*} F^{*}(H(\Lambda, 5))$ is again nef.

Example 4.4. Let $\Lambda=\Lambda_{1} \cup \cdots \cup \Lambda_{N}$ be a general arrangement of pencils in $\mathbf{P}^{\mathbf{2}}$; blowup all the points in the base loci to obtain $\mathbf{B P}^{2}$. Let $f_{i}: R_{i} \rightarrow \mathbf{P}^{\mathbf{1}}$ be 
any Riemann surface that branch covers $\mathbf{P}^{\mathbf{1}}$ having as branch locus for $f_{i}$ the set $\pi_{i}\left(\Lambda_{i}^{\prime}\right)=\left\{\pi_{i}\left(C_{i j}^{\prime}\right) \mid C_{i j} \in \Lambda_{i}\right\}$, where $\pi_{i}: \mathbf{B P}^{\mathbf{2}} \rightarrow \mathbf{P}^{\mathbf{1}}$ is the natural projection associated to the pencil $P_{i}$ belonging to $\Lambda_{i}, C_{i j}^{\prime}$ is the proper transform of $C_{i j}$, and $\Lambda_{i}^{\prime}=\left\{C_{i j}^{\prime} \mid C_{i j} \in \Lambda_{i}\right\}$.

Construct $X$ contained in $M:=R_{1} \times R_{2} \times \cdots \times R_{N} \times \mathbf{B P}^{2}$ by requiring that $X=\left\{\left(x_{1}, x_{2}, \ldots, x_{N}, p\right) \in M \mid \pi_{i}(p)=f_{i}\left(x_{i}\right)\right.$ for $\left.i=1, \ldots, N\right\} . X$ is a smooth surface. This follows from the fact that the matrix

$$
\left[\begin{array}{cccccc}
\frac{\partial f_{1}}{\partial x_{1}} & 0 & \cdots & 0 & -\frac{\partial \pi_{1}}{\partial x} & -\frac{\partial \pi_{1}}{\partial y} \\
0 & \frac{\partial f_{2}}{\partial x_{2}} & & 0 & -\frac{\partial \pi_{2}}{\partial x} & -\frac{\partial \pi_{2}}{\partial y} \\
0 & \cdots & \frac{\partial f_{i}}{\partial x_{i}} & 0 & -\frac{\partial \pi_{i}}{\partial x} & -\frac{\partial \pi_{i}}{\partial y} \\
0 & \cdots & \cdots & \frac{\partial f_{N}}{\partial x_{N}} & -\frac{\partial \pi_{N}}{\partial x} & -\frac{\partial \pi_{N}}{\partial y}
\end{array}\right]
$$

is of maximal rank. The left most $N$ by $N$ minor is of nonzero determinant at $\left(x_{1}, x_{2}, \ldots, x_{N}, p\right)$ unless $p \in C_{i j}^{\prime}$ for some $C_{i j}^{\prime} \in \Lambda^{\prime}$ and $\partial f_{i}\left(x_{i}\right) / \partial x_{i}=0$. In the case that the left most minor has determinant 0 , we have that since $\Lambda$ is a general pencil, at most two $C_{i j}^{\prime}$, say $C_{i j}^{\prime}$ and $C_{I J}^{\prime}$, can intersect at $p$ and they would meet transversely. Since $C_{i j}^{\prime}$ and $C_{I J}^{\prime}$ are nonsingular fibers of $\pi_{i}$ and $\pi_{I}$, respectively, one has that

$$
\operatorname{det}\left(\begin{array}{ll}
\frac{\partial \pi_{i}}{\partial x} & \frac{\partial \pi_{i}}{\partial y} \\
\frac{\partial \pi_{I}}{\partial x} & \frac{\partial \pi_{I}}{\partial y}
\end{array}\right) \neq 0 \quad \text { at } p .
$$

This compensates for the zero terms $\partial f_{i}\left(x_{i}\right) / \partial x_{i}$ and $\partial f_{I}\left(x_{I}\right) / \partial x_{I}$ and gives maximal rank $N$, so $X$ is smooth.

Now $X$ branch covers $\mathbf{B P}^{2}$, with $\pi: X \rightarrow \mathbf{B P}^{2}$ being the restriction to $X$ of the projection of $M$ onto the factor $\mathbf{B P}^{2}$, and $\pi$ is ramified precisely over $\Lambda^{\prime}$. This is seen by using the implicit function theorem in conjunction with the above $N \times(N+2)$ matrix. Since $\Lambda$ is a general arrangement of pencils, let $\pi_{1} \times \pi_{2} \times \cdots \times \pi_{k}: \mathbf{B P}^{\mathbf{2}} \rightarrow \mathbf{P}^{\mathbf{1}} \times \mathbf{P}^{\mathbf{1}} \times \cdots \times \mathbf{P}^{\mathbf{1}}$ be of maximal rank except at $E C$ union finitely many points. If $g\left(R_{i}\right) \geq 1$ for $i=1, \ldots, k$ then the Stein factorizations $S C_{i}$ (of $\pi_{i} \circ \pi$ ) factor through $R_{i}$ to $\mathbf{P}^{\mathbf{1}}$ giving that $g\left(S C_{i}\right) \geq g\left(R_{i}\right) \geq 1$ for $i=1, \ldots, k$. One then concludes by maximality of rank of $\pi_{1} \times \pi_{2} \times \cdots \times \pi_{k}$ that if $C_{j} \in \Lambda^{\prime}$ then $C_{j}$ must map onto say the $i$ th factor $\mathbf{P}^{1}$ for some $i=1, \ldots, k$. Hence each irreducible component $\widehat{C}_{j}$ of $\pi^{-1}\left(C_{j}\right)$ maps onto $S C_{i}$ over the $i$ th factor $\mathbf{P}^{1}$ and $e\left(\widehat{C}_{j}\right) \leq 0$. However, in order to guarantee that each $\widehat{C}_{j} \in \pi^{-1}(E C)$ has $e\left(\widehat{C}_{j}\right) \leq 0$, we must assume that $g\left(R_{i}\right) \geq 1$ for $i=1, \ldots, k, k+1, \ldots, N$. Since each $\widehat{C}_{j}$ has nonpositive self-intersection, all the hypotheses of Theorem 5 hold. We conclude that if each genus $g\left(R_{1}\right), g\left(R_{2}\right), \ldots, g\left(R_{N}\right)$ is positive then $T^{*} X$ is nef.

\section{ACKNOWLEDGMENT}

This work was partially supported by an OCSLA grant from East Carolina University. 


\section{REFERENCES}

1. G. Barthel, F. Hirzebruch, and T. Höfer, Geradenkonfigurationen und Algebraische Flächen: Eine Veroffentlichung des Max Planck Institut fur Mathematik, Bonn, Aspekte der Math., Ser. D, vol. 4, Vieweg \& Sohn, Braunschweig, 1987.

2. F. Hirzebruch, Arrangements of lines and algebraic surfaces, Arithmetic and Geometry, Vol. 2, Prog. Math., vol. 36, Birkhäuser, Boston, Basel, and Stuttgart, 1983, pp. 113-140.

3. T. Höfer, Ballquotienten als verzweigte uberlagegungen der projektiven ebene, Sonderforschungsbereich 40, MPI/SFB 85-23, Max Planck Institute, 1985.

4. A. Sommese, On the density of ratios of Chern numbers of algebraic surfaces, Math. Ann. 268 (1984), 207-221.

5. M. J. Spurr, Nef cotangent bundles over line arrangements, Manuscripta Math. 62 (1988), 369-374.

Department of Mathematics, East Carolina University, Greenville, North Carolina 27858

E-mail address: MAMMSPUR@ECUVM1.BITNET 